vergent with denomin:tor $q_{k}$ s.tisfying

VOL. VI DÉDIÉ À M. CASIMIR KURATOWSKI

$$
N<q_{k}<e N \quad\left(c<c_{0}, N<N_{0}\right)
$$

has a measure grecter th $\mathrm{n} 1-\varepsilon$

We $\operatorname{sh} 11$ return to this subject elsewhere.

\title{
REFERENOES
}

[1] P. Szüsz, Bemerkungen zur Approximation einer reelleǹ $Z$ xhl durch Brüche, Acta Mathematica Academiae Scientiarum Hungaricae 6 (1955), p. 203-212.

[2] P. Turán, Eine neue Methode in der Analysis und deren Anwendungen, Budapest 1953 (Eor an enlarged version seo the Cainese edition, Peking 1956).

Regu par la Rédaction le 16.11,195\%, en version modifiée le 10.6.1958

(a)

\section{ON THE APPROXIMATE SOLUTIONS OF FUNCTIONAL EQUATIONS IN $L^{p}$ SPACES}

$\mathrm{BY}$

M. ALTMAN (WARSAW)

In papers [1] and [2] we have suggested an iterative method for solving non-linear functional equations in Banach spaces. This method may also be regarded as a generalization of Newton's well-lknown classical method. But this generalization is essentially different from that given by L. V. Kantorovitch [5].

The present paper contains a specification for the case of the real $L^{p}$-spaces and a real Hilbert space of the iterative method defined in paper [2]. An application to approximate solutions of operator equations in this space is also given. In particular we consider in the $L^{p}$-spaces an analogue of the method of steepest descent for non-linear operator equations.

The iterative process for solving non-linear functional equations is defined in papers [1], [2] as follows:

Let $X$ be a Banach space and let $F(x), x \in X$, be a non-linear continuous functional which is differentiable in the sense of Fréchet. Then the approximate process for solving the non-linear functional equation

$$
F(x)=0
$$

is defined by the formula

$$
x_{1}=x_{0}-\frac{F\left(x_{0}\right)}{f_{0}\left(y_{0}\right)} y_{0}, \quad x_{n+1}=x_{n}-\frac{F\left(x_{n}\right)}{f_{n}\left(y_{n}\right)} y_{n},
$$

where $x_{0}$ is the initial approximate solution, $f_{n}=F^{\prime}\left(x_{n}\right)$ for $n=0,1,2, \ldots$ denotes the Fréchet differential of $F(x)$ as the point $x=x_{n}$, and $y_{n}$ are elements appropriately chosen in $X, \quad$. e. $\left\|y_{n}\right\|=1, f_{n}\left(y_{n}\right)=\left\|f_{n}\right\|$, $n=0,1,2, \ldots$, prorided that such a choice is possible.

The specification for the case of the real $L^{p}$-spaces and the real Hilbert space consists in the appropriate choice of the elements $y_{n}$. It appears that in this case the choice of the elements $y_{n}$ is effective and may be realized in a simple manner. 
Now let $X$ be a real Hilbert space and let $F(x)$ bo a non-linear continuous functional defined on the closed sphere $S\left(x_{0}, r\right)$ of $X$ with centre $x_{0}$ and radius $r$, having two continuous Fréchet derivatives.

Since the Fréchet differential $F^{\prime}(x)$ of $F(x)$ is a linear functional on the Hilbert space $X$, it can be represented in the form

$$
\dot{H}^{\prime}(x) y=(\Delta F(x), y), \quad y \in X,
$$

and the element $\Delta F(x)$ is called the gradient of $F$.

The iterative process (2) assumes here the form

(3) $\quad x_{1}=x_{0}-\frac{F\left(x_{0}\right)}{\left\|\Delta F\left(x_{0}\right)\right\|^{2}} \Delta W^{\prime}\left(x_{0}\right), \quad x_{n+1}=x_{n}-\frac{F\left(x_{n}\right)}{\left\|\Delta F\left(x_{n}\right)\right\|^{2}} \Delta F^{\prime}\left(x_{n}\right)$.

It is easy to see that such a choice of the elements $y_{n}=\Delta F\left(x_{n}\right)$ for $n=0,1,2, \ldots$ satisfies the condition of Theorem 1 of [2].

A convergence theorem of process (3) to a solution of equation (1) is given by the general case considered in paper $[2\rfloor$ (Theorem 1 ).

Now let $X$ be the real space $L^{p}=L^{p}(a, b)$ with $p>1$; then the conjugate space of $L^{p}$ is $L^{q}$, where $1 / p+1 / q=1$.

Let $f \epsilon L^{q}$ be an arbitrary linear functional on $L^{p}$. Putting

$$
g(t)=\operatorname{sign} f(t)|f(t)|^{1 / p-1} \quad(a \leqslant t \leqslant b),
$$

we have

$$
\int_{a}^{b} f(t) g(t) d t=\|f\|^{Q}
$$

Thus, for $\bar{g}=g /\|g\|$ we obtain $\bar{g} \epsilon L^{p},\|g\|=1$, and it is easy to see that the norm of the functional $f$ is reached at the element $\bar{g} \in L^{p}, i . e$.

$$
\int_{a}^{b} f(t) \bar{g}(t) d t=\|f\| \text {. }
$$

As in the case of the Hilbert space, let us assume that $F(x)$ is a non-linear continuous functional defined on the closed sphere $S\left(x_{0}, r\right)$, having two Fréchet derivatives.

Since the Fréchet differential $F^{\prime}(x)$ of $F(x)$ is a linear functional on $L^{p}$, we put

$$
g(x)(t)=\operatorname{sign}{F^{\prime}}^{\prime}(x)(t)\left|F^{\prime}(x)(t)\right|^{1 /(p-1)}
$$

and obtain, by (4) and (5),

$\therefore \quad \int_{a}^{b} F^{\prime}(x)(t) g(x)(t) d t=\left\|F^{\prime}(x)\right\|^{q}, \quad \int_{a}^{b} F^{\prime}(x)(t) \bar{g}(x)(t) d t=\left\|F^{\prime}(x)\right\|$,

where $\bar{g}(x)=g(x) /\|g(x)\|$

Iterative process (3) assumes here the form

$$
\text { (7) } \quad x_{1}=x_{0}-\frac{F\left(x_{0}\right)}{\left\|F^{\prime}\left(x_{0}\right)\right\|^{\alpha}} g\left(x_{0}\right), \quad x_{n+1}=x_{n}-\frac{F\left(x_{n}\right)}{\left\|F^{\prime}\left(x_{n}\right)\right\|^{q}} g\left(x_{n}\right) \text {, }
$$

where $g\left(x_{n}\right)$ for $n=0,1,2, \ldots$ is defined by formula $(6)$.

It is easy to see that such a choice of the elements $y_{n}=g\left(x_{n}\right)$ satisfies the above mentioned condition of Theorem 1 of [2].

Thus, Theorem 1 of [2] gives also a convergence theorem of process

(7) to a solution of equation (1).

Paper [3] contains an application of Newton's method of solving non-linear functional equations (1), defined by process (3), to the approximate solution of operator equations

$$
P(x)=0
$$

in $L^{p}$-space. It is shown in [3] that equation (8) can be reduced to equation (1) by setting $F^{\prime}(x)=\|P(x)\|^{p}=0$.

Thus we obtain in [3] the following approximate process for solving operator equation (8):

$$
x_{1}=x_{0}-\frac{\left\|P\left(x_{0}\right)\right\|^{p}}{p\left\|f\left(x_{0}\right)\right\|^{q}} y_{0}, \quad x_{n+1}=x_{n}-\frac{\left\|P\left(x_{n}\right)\right\|^{p}}{p\left\|f\left(x_{n}\right)\right\|^{q}} y_{n},
$$

where

$$
f(x)=\bar{P}^{\prime}(x)\left(\operatorname{sign} P(x)(t)|P(x)(t)|^{p-1}\right) \epsilon L^{q},
$$

$P^{\prime}(x)$ denotes the Fréchet differential of $P(x), \bar{P}^{\prime}(x)$ is the adjoint of $P^{\prime}(x)$ and

$$
y_{n}=y_{n}(t)=\operatorname{sign} f\left(x_{n}\right)\left|f\left(x_{n}\right)(t)\right|^{1 / p-1} \quad(n=0,1,2, \ldots) .
$$

On the other hand, operator equation (8) can also be reduced to functional equation (1) by setting $F(x)=\|P(x)\|=0$.

Applying process (7) to this case we obtain the following iterative process of solving operator equation (8):

$$
x_{1}=x_{0}-\frac{\left\|P\left(x_{0}\right)\right\|^{p}}{\left\|f\left(x_{0}\right)\right\|^{\alpha}} y_{0}, \quad x_{n+1}=x_{n}-\frac{\left\|P\left(x_{n}\right)\right\|^{p}}{\left\|f\left(x_{n}\right)\right\|^{\alpha}} y_{n},
$$

where $f\left(x_{n}\right)$ and $y_{n}$ have the same meaning as above.

Notice that we use here a result of S. Mazur [6] concerning the differentiability in Fréchet's sense of the norm $\|x\|$ in $L^{p}$ to compute the Fréchet differential $F^{\prime}(x)$ of $F(x)=\|P(x)\|$ provided that operator $P(x)$ is differentiable in Fréchet's sense.

Colloquium Mathematicum vi 
It should also be remarked that in the case of a real Hilbert sface, as it is shown in [4], process (10) may be regarded as an extension of the method of steepest descent.

We shall now give a convergence theorem of process (10) to a solution of operator equation (8).

Let $S\left(x_{0}, r\right)$ be a closed sphere in $L^{p}$ with centre $x_{0}$ and radius $r$. Consider the non-linear operator equation (8), where $P$ is a non-linear continuous operator defined on the sphere $S\left(x_{0}, r\right)$ with values in $L^{p}$ with $1<p$.

Let us assume that $P(x)$ is differentiable in the sense of Fréchet in the sphere $S\left(x_{0}, r\right)$.

We suppose also that there exists the Fréchet differential $f^{\prime}(x)$ of $f(x)$, where $f(x)$ is defined by formula (9) and is bounded in the sphere $S\left(x_{0}, r\right)$, i. e., that there exists a constant $K$ such that

$$
\left\|f^{\prime}(x)\right\| \leqslant K \quad \text { for every } \quad x \text { of } S\left(x_{0}, r\right) .
$$

Sufficient conditions for the existence of a solution of equation (8) as well a convergence theorem of process (10) to this solution are given by the following

THEOREM 1. Let us assume that the following conditions are satisfied: $1^{\circ}$ the Fréchet differential $P^{\prime}(x)$ of $P(x)$ exists and satisfies condition

$$
\frac{1}{\left\|f\left(x_{0}\right)\right\|} \leqslant B_{0}
$$

$2^{\circ}$ the Fréchet differential $f^{\prime}(x)$ of $f(x)$ exists and satisfies condition (11) $3^{\circ}$ the first approximate solution satisfies the inequality

$$
\left\|x_{1}-x_{0}\right\|=\frac{\left\|P\left(x_{0}\right)\right\|^{p}}{\left\|f\left(x_{0}\right)\right\|} \leqslant \eta_{0},
$$

where $\eta_{0}$ is a constant;

$4^{0}$ the consiants $B_{n}, \eta_{0}$ and $K$ are subjected to the condition

and

$$
p B_{0} K \eta_{0}=h_{0} \leqslant \frac{1}{2}
$$

$$
r=\frac{1-\sqrt{1-2 h_{0}}}{h_{0}} \eta_{0} .
$$

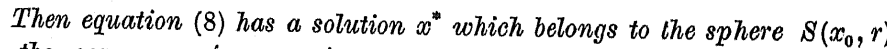
and the sequence of approximate solutions $x_{n}$ defined by process $(10)$ converges to $x$.
For the error estimate we have the formula

$$
\left\|x_{n}-x^{*}\right\| \leqslant \frac{1}{2^{n-1}}\left(2 h_{0}\right)^{2^{n}-1} \eta_{0}
$$

Proof. Multiplying equations (10) by $f\left(x_{n}\right)$ and integrating we obtain

$$
\begin{aligned}
\left\|P\left(x_{n}\right)\right\|^{p} & =\int_{a}^{b} f\left(x_{n}\right)(t)\left(x_{n}-x_{n+1}\right)(t) d t \\
& =\int_{a}^{b} \operatorname{sign} P\left(x_{n}\right)(t)\left|P\left(x_{n}\right)(t)\right|^{p-1} P^{\prime}\left(x_{n}\right)\left(x_{n}-x_{n+1}\right)(t) d t .
\end{aligned}
$$

Thus we have

$$
-\left\|P\left(x_{n}\right)\right\|^{p}=\int_{a}^{b} f\left(x_{n}\right)(t)\left(x_{n+1}-x_{n}\right)(t) d t .
$$

Hence we get

$$
\begin{aligned}
\left\|P\left(x_{n}\right)\right\|^{p}= & \left\|P\left(x_{n}\right)\right\|^{p}-\left\|P\left(x_{n-1}\right)\right\|^{p}-\int_{a}^{b} f\left(x_{n-1}\right)(t)\left(x_{n}-x_{n-1}\right)(t) d t \\
= & \left\|P\left(x_{n}\right)\right\|^{p}-\left\|P\left(x_{n-1}\right)\right\|^{p}-p \int_{a}^{b} f\left(x_{n-1}\right)(t)\left(x_{n}-x_{n-1}\right)(t) d t+ \\
& +(p-1) \int_{a}^{b} f\left(x_{n-1}\right)(t)\left(x_{n}-x_{n-1}\right)(t) d t .
\end{aligned}
$$

Thus, we have by (17)

(18) $\quad\left\|P\left(x_{n}\right)\right\|^{p} \leqslant\left\|P\left(x_{n}\right)\right\|^{p}-\left\|P\left(x_{n-1}\right)\right\|^{p}-p \int_{a}^{b} f\left(x_{n-1}\right)(t) \cdot\left(x_{n}-x_{n-1}\right)(t) d t$.

Using the analogue of Taylor's formula we 'have by (18) and (11)

$$
\left\|P\left(x_{n}\right)\right\|^{p} \leqslant \frac{p K}{2}\left\|x_{n}-x_{n-1}\right\|^{2} .
$$

Since $\left\|y_{n}\right\|=\left\|f\left(x_{n}\right)\right\|^{q / p}$, we have by (10)

$$
\left\|x_{n+1}-x_{n}\right\|=\frac{\left\|P\left(x_{n}\right)\right\|^{p}}{\left\|f\left(x_{n}\right)\right\|} .
$$

Thus we obtain by (19) and (20)

$$
\left\|x_{n+1}-x_{n}\right\|_{1} \leqslant \frac{p K}{2\left\|f\left(x_{n}\right)\right\|}\left\|x_{n}-x_{n-1}\right\|^{2} .
$$


We shall now estimate the norm $\left\|f\left(x_{1}\right)\right\|$ :

$$
\left\|f\left(x_{1}\right)\right\| \geqslant\left\|f\left(x_{0}\right)\right\|-\left.\right|_{1} f\left(x_{0}\right)-f\left(x_{1}\right)\|=\| f\left(x_{0}\right) \|\left(1-\frac{\left\|f\left(x_{0}\right)-f\left(x_{1}\right)-f\left(x_{1}\right)\right\|}{\left\|f\left(x_{0}\right)\right\|}\right) .
$$

Hence, using the analogue of Lagrange's formula, we have by (12) and (11).

$\left\|f\left(x_{1}\right)\right\| \geqslant\left\|f\left(x_{0}\right)\right\| \cdot\left(1-B_{0} K \eta_{0}\right)>\left\|f\left(x_{0}\right)\right\| \cdot\left(1-p B_{0} K \eta_{0}\right)=\left\|f\left(x_{0}\right)\right\|\left(1-h_{0}\right)$.

Thus, we obtain

$$
\left\|f\left(x_{1}\right)\right\|^{-1} \leqslant \frac{1}{1-h_{0}} \cdot \frac{1}{\left\|f\left(x_{0}\right)\right\|} \leqslant \frac{B_{0}}{1-h_{0}}=B_{1} .
$$

For $n=1,(21)$ gives by (13) and (22)

$$
\left\|x_{2}-x_{1}\right\| \leqslant \frac{1}{2} \cdot \frac{B_{0}}{1-h_{0}} p K \eta_{0}^{2}=\frac{1}{2} \cdot \frac{h_{0} \eta_{0}}{1-h_{0}}=\eta_{1} .
$$

Condition (14) is satisfied for $x=x_{1}$ :

(24) $h_{1}=p B_{1} K \eta_{1}=p \cdot \frac{B_{0}}{1-h_{0}} \cdot \frac{K}{2} \cdot \frac{h_{0} \eta_{0}}{1-h_{0}}=\frac{h_{0}^{2}}{2\left(1-h_{0}\right)^{2}} \leqslant 2 h_{0}^{2} \leqslant \frac{1}{2}$.

Thus, conditions $1^{\circ}-4^{\circ}$ are satisfied for $x=x_{1}$ replacing the numbers $B_{0}, \eta_{0}$ and $h_{0}$ by $B_{1}, \eta_{1}$ and $h_{1}$ defined by $(22),(23)$ and (24). Hence we can define by induction the approximate solution $x_{n}$ and the corresponding numbers $B_{n}, \eta_{n}$ and $h_{n}$, which satisfy the formulas analogous to (13), (22), (23) and (24):

$$
\begin{gathered}
\left\|x_{n+1}-x_{n}\right\| \leqslant \eta_{n}, \\
B_{n}=\frac{B_{n-1}}{1-h_{n-1}}, \\
\eta_{n}=\frac{1}{2} \cdot \frac{h_{n-1} \eta_{n-1}}{1-h_{n-1}}, \\
h_{n}=\frac{1}{2} \frac{h_{n-1}^{2}}{\left(1-h_{n-1}\right)^{n}} .
\end{gathered}
$$

Using the same argument as in paper [2] we obtain from $\left(13^{\prime}\right),\left(22^{\prime}\right)$ $\left(23^{\prime}\right)$ and $\left(24^{\prime}\right)$

$$
\left\|x_{n+p}-x_{n}\right\| \leqslant \frac{1}{2^{n-1}}\left(2 h_{0}\right)^{2^{n}-1} \eta_{0}
$$

The same argument shows that all the approximate solutions $x_{n}$ are contained in the sphere $S\left(x_{0}, r\right)$ defined by (15). It follows from $(25)$ that the sequence $\left(x_{n}\right)$ converges to an element $x^{*}$ of the sphere $S\left(x_{0}, r\right)$. It follows from (19) that $x^{*}$ is a solution of equation (8).

Formula (17) results trom (25).

Remark 1. Condition (14) can be replaced by the following:

$$
h_{0}=p B_{0}^{2} K D \leqslant \frac{1}{2},
$$

where $D$ is a constant such that $\left\|P\left(x_{0}\right)\right\|^{p} \leqslant D$.

Remark 2. It is convenient to verify that condition (11) is satisfied in a constant sphere containing the sphere $S\left(x_{0}, r\right)$ where the radius $r$ is defined by (15). For this purpose we can take, for instance, the sphere $S\left(x_{0}, r\right)$ where $r=2 \eta_{0}$ or $r=2 B_{0} D$.

If $X$ is a real Hilbert space and $P$ is a non-linear operator operating in this space and defined on the sphere $S\left(x_{0}, r\right)$, then the process (10) is of the form (see [3])

(26) $\quad x_{1}=x_{0}-\frac{\left\|P\left(x_{0}\right)\right\|^{2}}{\left\|Q\left(x_{0}\right)\right\|^{2}} Q\left(x_{0}\right), \quad x_{n+1}=x_{n}-\frac{\left\|P\left(x_{n}\right)\right\|^{2}}{\left\|Q\left(x_{n}\right)\right\|^{2}} Q\left(x_{n}\right)$,

where $Q(x)=P^{\prime}(x) \bar{P}(x)$.

Suppose that there exists the Fréchet differential $Q^{\prime}(x)$ of $Q(x)$ and that $Q^{\prime}(x)$ is bounded in the sphere $S\left(x_{0}, r\right), i . e$. , that there exists a constant $K$ such that

$$
\left\|Q^{\prime}(x)\right\| \leqslant K \quad \text { for every } \quad x \text { of } S\left(x_{0}, r\right) .
$$

As a particular case of Theorem 1 we have

THEOREM 2. Let us assume that the following conditions are satisfied:

$1^{0}$ The Fréchet differential $P^{\prime}(x)$ exists in the sphere $S\left(x_{0} r\right)$ and satisfies the condition

$$
\frac{1}{\| Q\left(x_{0} \|\right.} \leqslant B_{0}
$$

$2^{\circ}$ The Fréchet differential $Q^{\prime}(x)$ of $Q(x)$ exists and satisfies the condition (27)

$3^{\circ}$ The first approximate solution satisfies the inequality

$$
\left\|x_{1}-x_{0}\right\|=\frac{\left\|P\left(x_{0}\right)\right\|^{2}}{\left\|Q\left(x_{0}\right)\right\|} \leqslant \eta_{0}
$$

$4^{\circ}$ The constants $B_{0}, \eta_{0}$ and $K$ are subjected to condition (14) with $p=2$ and $r$ is defined by (15).

Then equation (8) has a solution which belongs to sphere $S\left(x_{0}, r\right)$ and the sequence of the approximate solutions $x_{n}$ converges to $x$. 
For the error estimate we have the formula (16).

Remark 3. Remarks 1 and 2 are also applicable to Theorem 2 .

\section{REFERENOES}

[1] M. Altman, On the approximate solutions of non-linear functional equations, Bulletin de l'Académie Polonaise des Sciences, Classe III, 5 (1957), p. 457-460.

[2] - Ooncerning approximate solutions of non-linear functional equations, ibidem 5 (1957), p. 461-465.

[3] - On the approximate solutions of operator equations in $L^{p-s p a c e s, ~ i b i d e m ~}$ (1957), p. 1099.1103.

[4] - Connection between the method of steepest descent and Newton's method, ibidem 5 (1957), p. 1031-1036.

[5] Л. В. Канторович, $O$ методе Нroтона, Труды Математического Института им. Б. А. Стеклова 28 (1949), p. 101-144.

[6] S. Mazur, Über schwache Konvergenz in den Räumen $L^{p}$, Studia Mathematica 4 (1933), p. 128-133.

MATHEMATICAL INSTITUTE OF THE POLISH ACADEMY OF SCIENCES

Resu par la Rédaction le 1\% 11. $195 \%$

\section{ON CANTOR'S PRODUCTS \\ BY}

A. RENYI (BUDAPEST)

G. Cantor [4] (see also [16], p. 122-127) considered the representation of a real number $x>1$ in the form of the infinite product

$$
x=\prod_{n=1}^{\infty}\left(1+\frac{1}{q_{n}}\right)
$$

where $q_{n}=q_{n}(x)$ is a sequence of positive integers, which may be defined as follows: we choose for $q_{1}$ the least positive integer for which $1+1 / q_{1} \leqslant x$ and if $q_{1}, q_{2}, \ldots, q_{n-1}$ are already chosen, we choose for $q_{n}$ the least positive integer for which $\prod_{n}^{n}\left(1+1 / q_{k}\right) \leqslant x$. Clearly if $x$ is contained in the interval $2^{k-1}<x \leqslant 2^{k} \quad(k=1,2 \ldots), \quad$ then $\quad q_{1}=q_{2}=\ldots=q_{k-1}=1, \quad$ and $1<x / \prod_{j=1}^{k-1}\left(1+1 / q_{j}\right) \leqslant 2$. Thus we may restrict ourselves to the values of $x$ lying in the interval $1<x \leqslant 2$. In this case clearly

$$
q_{n+1} \geqslant q_{n}^{2} \quad(n=1,2, \ldots) .
$$

Let us put

$$
E_{0}(x)=x, \quad E_{n}(x)=x / \prod_{k=1}^{n}\left(1+\frac{1}{q_{k}}\right) \quad(n=1,2, \ldots) .
$$

It is easy to see that if $x$ is rational, $x=a / b$ where $a$ and $b$ are positive integers, $b<a \leqslant 2 b$, then we obtain by the algorithm described above a finite representation for $x$ of the form

$$
\frac{a}{b}=\prod_{n=1}^{N}\left(1+\frac{1}{q_{n}}\right)
$$

since putting $E_{n}(a / b)=a_{n} / b_{n}$ we have $a_{n+1}-b_{n+1}<a_{n}-b_{n}$; it follows that $N \leqslant a-b$ 